\title{
Newly appointed editors
}

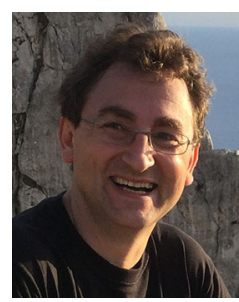

Adrian Langer born in 1973, studied mathematics at the University of Warsaw, where he obtained his PhD in 1998. Afterwards he held postdoctoral positions at the ICTP in Trieste (1999) and the University of Warwick (2000-2002). He was a visiting professor at the MPI, Bonn (2007) and the Universität Duisburg-Essen (2011-2012, supported by a Bessel Research Award from the Alexander von Humboldt Foundation). He was appointed to various positions at the University of Warsaw (since 1994) and the Institute of Mathematics of the Polish Academy of Sciences (2004-2012). He obtained his habilitation in 2004 at the University of Warsaw and a Polish state professorship in 2012. His research interests include algebraic geometry and to a lesser extent arithmetic geometry and commutative algebra.

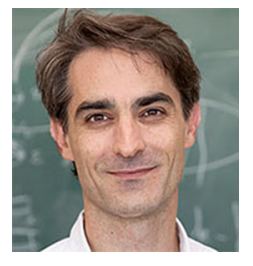

Stéphane Sabourau studied mathematics at the École normale supérieure de Lyon and received his $\mathrm{PhD}$ in mathematics at the University of Montpellier in 2001. From 2002 to 2011, he was an assistant professor at the University of Tours, where he obtained his habilitation. Since 2011, he has been a professor at the University Paris-Est Créteil, where he served as the director of the mathematics research department from 2015 to 2019. His research interests lie in the area of metric geometry and quantitative topology, including global Riemannian geometry, hyperbolic geometry, geometric calculus of variations, geometric group theory, systolic geometry, and asymptotic geometry.

Publisher's Note Springer Nature remains neutral with regard to jurisdictional claims in published maps and institutional affiliations. 